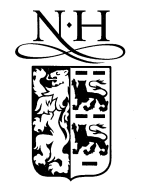

\title{
Experimental demonstration of negative dispersion without absorption $^{1}$
}

\author{
A. Wicht ${ }^{\mathrm{a}, 2}$, M. Müller ${ }^{\mathrm{a}}$, R.-H. Rinkleff ${ }^{\mathrm{a}, *}$, A. Rocco ${ }^{\mathrm{b}}$, K. Danzmann ${ }^{\mathrm{a}, \mathrm{b}}$ \\ ${ }^{a}$ Institut für Atom- und Molekülphysik, Abteilung Spektroskopie, Universität Hannover, Callinstr. 38, D 30167 Hannover, Germany \\ b Max-Planck-Institut für Quantenoptik, Außenstelle Hannover, Callinstr. 38, D 30167 Hannover, Germany
}

Received 1 July 1999; received in revised form 6 September 1999; accepted 10 September 1999

\begin{abstract}
We present the results of an experiment proving in principle that negative (anomalous) dispersive transparent media can be realized. The Ca-transition $4 \mathrm{~s}^{21} \mathrm{~S}_{0} \rightarrow 4 \mathrm{~s} 4 \mathrm{p}^{1} \mathrm{P}_{1}$ at $\lambda=423 \mathrm{~nm}$ was chosen to investigate the absorption and index of refraction spectra of a two-level system strongly driven at resonance. For the simultaneous high precision measurement of loss and phase shift imposed on a probe field during the interaction with a chopped Ca-atomic beam we used a novel spectrometer which is based on a phase modulated Mach-Zehnder interferometer. So far, a dispersion of $\partial_{\omega} n_{0}=-(3.1 \pm$ $1.0) \times 10^{-10} / \mathrm{MHz}$ accompanied by residual absorption of $\alpha_{0}=(0.125 \pm 0.040) \% / \mathrm{cm}$ has been achieved. These values are compared to the requirements which have to be achieved for a certain application of negative dispersion without absorption, that is for a broadband high build-up cavity (white-light cavity). (C) 2000 Elsevier Science B.V. All rights reserved.
\end{abstract}

Keywords: Atomic coherence effects; Negative (anomalous) dispersion; Phase modulated Mach-Zehnder interferometer

Over the last decade, novel optical media based on atomic coherence and interference effects have gained much attention. A variety of new effects was predicted to result from the application of these ideas to gaseous or even solid media [1], some of which have already been demonstrated experimentally. The pioneering work of Kocharovskaya [2], Harris [3], and Scully [4] initiated the investigation of lasing without inversion (LWI) both theoretically [5] and experimentally [6]. LWI may provide a possibility to

\footnotetext{
* Corresponding author.

${ }^{1}$ Dedicated to Prof. Dr. Marlan O. Scully on the occasion of his 60-th birthday.

${ }^{2}$ E-mail address: wicht@pallas.amp.uni-hannover.de.
}

realize sources of coherent radiation at UV or even $\mathrm{X}$-ray wavelength [7]. It has also been shown theoretically [8] and experimentally [9] that it is possible to enhance the index of refraction of certain systems at optical frequencies, where the absorption is strongly suppressed or even canceled. This may be of interest for those applications where strongly refractive media are essential, as for example in optical immersion microscopy [10]. It is clear that atomic or molecular media featuring an ultra-large index of refraction will also exhibit very strong dispersion $\left(\partial_{\omega} n\right)$, since a large index of refraction is always confined within a frequency bandwidth of the order of an atomic or molecular linewidth. For example, in strongly positive (normal) dispersive transparent media $\left(\partial_{\omega} n \gg\right.$ 
$\left.1 / \omega_{0}\right)$, the propagation of light pulses seems to be 'frozen', since the group velocity can be reduced by several orders of magnitude (down to $17 \mathrm{~m} / \mathrm{s}$ so far [11]). Further, strongly positive dispersive media can be used to realize ultra-sensitive magnetometers [12] or to set up low-finesse but narrow-linewidth optical cavities [13]. The latter may serve as 'real' mechanical frequency standards for the optical domain, since the influence of cavity length fluctuations on the determination of the cavity resonance frequency can be suppressed relative to the influence of the fluctuations of the probing laser frequency.

In contrast to this, negative (anomalous) dispersive transparent media exhibiting just the right amount of dispersion could allow the realization of so-called white-light cavities featuring simultaneously high internal build-up and large bandwidth [14]. These cavities may in principle be useful for example for enhancing the sensitivity of interferometric gravitational wave detectors. In complementarity to the intra-cavity operation of strongly positive dispersive media, anomalous dispersive media introduced in an optical cavity could be used to realize a 'real' optical distance measurement device: since the spatial phase of an optical field with angular frequency $\omega=\omega_{0}+\delta$ is given by $\varphi=$ $\omega n(\omega) / c_{0} z$ with $z$ being a certain position along the axis of propagation, it no longer depends on frequency fluctuations, if $\omega \cdot n(\omega)=$ const within some frequency bandwidth centered at the point of operation $\omega_{0}$. As we will see later on, $n\left(\omega_{0}\right)=1$ may be assumed, so that the above mentioned requirement is achieved, if $\partial_{\omega_{0}} n=-1 / \omega_{0}$. In this case optical fields at all frequencies close to $\omega_{0}$ will exhibit the same wavelength, so that the dependence of the vacuum wavelength on frequency is compensated. Therefore, an optical cavity operated with an anomalous dispersive medium inside can be simultaneously resonant for all of these frequencies provided that it is resonant for, say, $\omega_{0}$. In this way, high-finesse but nevertheless broadband response cavities could be realized. The realization of negative dispersive transparent media is the main subject of this paper.

The most familiar example of a negative dispersive medium is a 'passive' (no coherent driving fields) atomic or molecular medium probed right in the very vicinity of a resonance. However, it is well known that this is accomplished by very strong absorption, so that such media are of no use for our purpose. Nevertheless, there are several more complicated configurations which are based on atomic coherence effects and which can provide the desired optical properties, for example, the double- $\Lambda$ scheme [14] or the strongly driven two-level system [15]. Among those, the latter seems to be most convenient from the experimental point of view [16]. In this paper we present a proof of principle experiment demonstrating for the first time that negative dispersion without absorption is feasible at optical frequencies. It is based on the optical properties of the Ca-transition $4 \mathrm{~s}^{2}{ }^{1} \mathrm{~S}_{0} \rightarrow 4 \mathrm{~s} 4 \mathrm{p}^{1} \mathrm{P}_{1}$ at $\lambda=423 \mathrm{~nm}$, which is strongly driven at resonance by a coherent driving field.

In the following, we will briefly summarize those aspects of strongly driven two-level systems which are of major importance within the context of negative dispersion without absorption. The experimental setup is presented, that is a diode laser based spectrometer which employs a novel spectroscopic method. The discussion of the experimental results clearly demonstrates that negative dispersion without absorption has in principle been achieved. Finally, we compare the dispersion and reduction of absorption achieved so far with the requirements for an application in a white-light cavity.

Since the pioneering work of Rautian and Sobel' man [17] and Mollow [18], a large number of papers have dealt with several aspects of strongly driven two-level atoms (TLA), both theoretically and experimentally [19]. However, there have been no experiments aimed at the simultaneous investigation of the absorption and the refractive index spectra within the central resonance of a strongly driven TLA in the optical domain. The spectrum of a TLA with resonance frequency $\omega_{0}=2 \pi \nu_{0}$ and natural linewidth $\gamma$, which is strongly driven at resonance by a coherent driving field $\mathscr{E}=E_{0} \cos \left(\omega_{0} t\right)$ of Rabi-frequency $\Omega=E_{0} \mathscr{D} /(2 \hbar)$ coupled to the atom by the electric dipole moment $\mathscr{D}$ can be calculated [15,21]. These spectra are given in Fig. 1, where the absorption and index of refraction for a weak probe field of frequency $\omega$ versus the probe field detuning $\Delta \omega=\omega$ $-\omega_{0}$ are shown. The figure illustrates the special case of the Ca-transition $4 \mathrm{~s}^{2}{ }^{1} \mathrm{~S}_{0} \rightarrow 4 \mathrm{~s} 4 \mathrm{p}^{1} \mathrm{P}_{1}$ at $\lambda=$ $423 \mathrm{~nm}$. The natural linewidth of this transition 

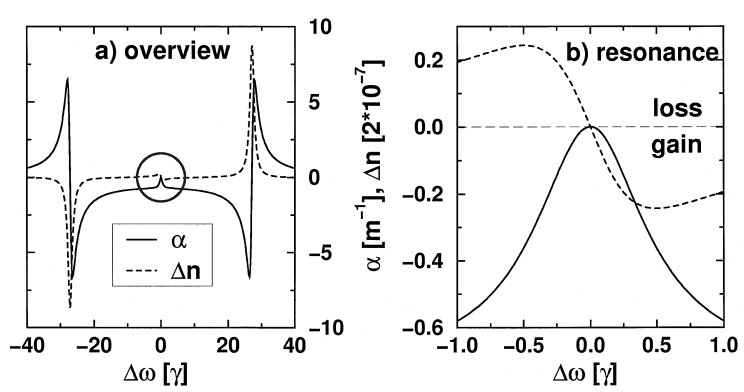

Fig. 1. Absorption and index of refraction profiles of a two-level system strongly driven at resonance versus probe laser frequency. The Ca-transition $4 \mathrm{~s}^{2}{ }^{1} \mathrm{~S}_{0} \rightarrow 4 \mathrm{~s} 4 \mathrm{p}^{1} \mathrm{P}_{1}$ at $\lambda=423 \mathrm{~nm}$ is shown. Natural linewidth $\gamma /(2 \pi)=34 \mathrm{MHz}$. An atomic density of $N=$ $5 \times 10^{10} \mathrm{~cm}^{-3}$ is assumed. The atoms are driven at atomic resonance by a coherent optical field at Rabi-frequency $\Omega=13.5 \gamma$.

given by Kisters et al. is $\gamma /(2 \pi)=34 \mathrm{MHz}$ [20]. For the plots, an atomic density of $N=5 \times$ $10^{10} \mathrm{~cm}^{-3}$ and a driving field Rabi-frequency of $\Omega=13.5 \gamma$ is assumed. Apart from the two Rabisidebands located at detunings $\Delta \omega \approx \pm 2 \Omega$, we find that at the central resonance $|\Delta \omega| \ll \gamma$ coinciding with the bare atomic resonance, the spectrum displays negative dispersion $\partial_{\omega} n<0$ at frequencies where the absolute value of the absorption $|\alpha|$ is strongly reduced. Although not apparent from Fig. 1, we find some very small residual loss at the exact resonance $\Delta \omega=0$. However, this may be neglected in applications as long as additional losses, for example round-trip losses of an empty white-light cavity, exceed this residual loss. From the theory of the strongly driven TLA we can calculate the absorption $\alpha_{0}$ and the dispersion $\partial_{\omega} n_{0}$ at resonance [15]:

$$
\begin{aligned}
& \alpha_{0}=\frac{\omega_{0}}{c_{0}} \cdot N \frac{3 \pi c_{0}^{3}}{\omega_{0}^{3}} \cdot \frac{1}{\left[1+8(\Omega / \gamma)^{2}\right]^{2}}, \\
& \partial_{\omega} n_{0}=-\frac{1}{\gamma} \cdot N \frac{3 \pi c_{0}^{3}}{\omega_{0}^{3}} \cdot \frac{1+32(\Omega / \gamma)^{4}}{\left[1+8(\Omega / \gamma)^{2}\right]^{3}} .
\end{aligned}
$$

These quantities are shown in Fig. 5 versus driving field Rabi-frequency $\Omega$. As can be seen in the limit of strong driving field, residual absorption is more strongly suppressed than the dispersion. Actually, it is this quality of the TLA which allows us to conclude that negative dispersion without absorption is feasible. In principle, for any application some very small residual absorption is acceptable, as mentioned above. Given this maximum tolerable loss we can always find some convenient driving field Rabifrequency $\Omega$ and some convenient atomic density $N$, for which the residual absorption $\alpha_{0}$ may be neglected and the desired amount of negative dispersion is achieved. To compare different systems with respect to their capability of providing negative dispersion without absorption, a figure of merit $\Theta_{\mathrm{FOM}}$ can be defined according to

$$
\Theta_{\mathrm{FOM}}=\frac{\partial_{\omega} n_{0}}{\left(-1 / \omega_{0}\right) \alpha_{0}} \stackrel{\text { TLA }}{=} \frac{c_{0}}{\gamma} \cdot \frac{1+32(\Omega / \gamma)^{4}}{1+8(\Omega / \gamma)^{2}} \text {. }
$$

$\Theta_{\text {FOM }}$ gives the ratio of the dispersion to the residual absorption at resonance, where the dispersion is normalized to that value which is needed to compensate for the variation of vacuum wavelength with frequency.

We set up an experiment in order to demonstrate negative dispersion without absorption experimentally and to investigate those conditions under which large figures of merit can be achieved. The experimental setup is shown in Fig. 2. Two identical laser systems, each based on an injection locked masterslave system and external cavity SHG (Second Harmonic Generation) are used as drive and probe lasers. The master oscillators are laser diodes SDL5410-C with optical feedback from a holographic grating (1400 l/mm) in Littrow-configuration. For the slaves we use SDL5420-C laser diodes capable of providing up to $\sim 150 \mathrm{~mW}$ of fundamental power at 846 $\mathrm{nm}$. The output from each of the slave lasers is frequency-doubled in a single resonant external cavity using $\mathrm{KNbO}_{3}$-crystals of length $9 \mathrm{~mm}$ and $15 \mathrm{~mm}$ for the probe and drive field respectively. About 20 $\mathrm{mW}$ of blue power at $423 \mathrm{~nm}$ are provided by the driving laser system. For more detailed information on the laser system the reader is referred to [22].

The drive laser is locked to the Ca-transition $4 \mathrm{~s}^{2} \mathrm{~S}_{0} \rightarrow 4 \mathrm{~s} 4 \mathrm{p}^{1} \mathrm{P}_{1}$ at $\lambda=423 \mathrm{~nm}$ via FM-spectroscopy (FMS) performed on a Ca-heatpipe. Some fraction of the output of the master oscillator of the drive laser is frequency shifted by $f_{\mathrm{FS}}=180.000$ $\mathrm{MHz}$ (FS) and is then used for phase-locking the master oscillator of the probe laser to the master of the drive at a tunable offset frequency $80 \mathrm{MHz}<$ 


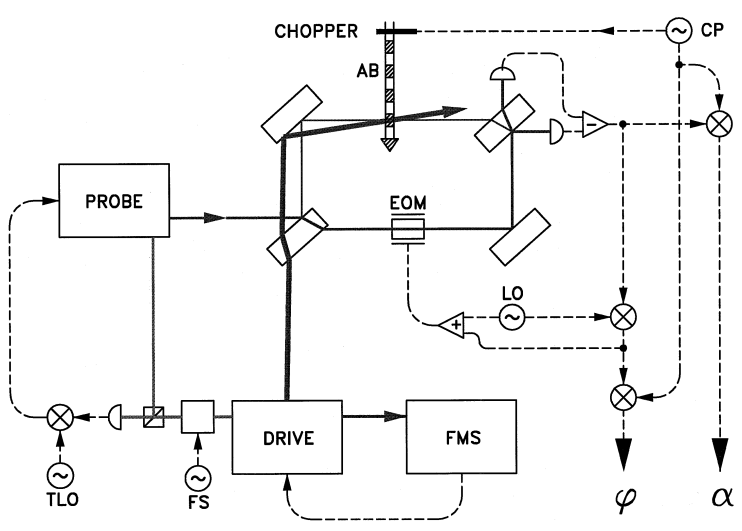

Fig. 2. Experimental setup. Two identical laser systems, each based on an injection locked master-slave system and external cavity SHG, are used as drive and probe lasers. The drive laser is locked to the Ca-transition via a FM-spectroscopy method (FMS). The probe laser is phase-locked to the drive laser at a tunable offset frequency (TLO). The probe laser enters a Mach-Zehnder interferometer. A fraction of the probe power intersects a chopped atomic beam $\left(\mathrm{AB}, \mathrm{EOM}, f_{\mathrm{CP}} \approx 1770 \mathrm{~Hz}\right)$ in the test path of the interferometer. The reference path is phase modulated (AB, EOM, $\left.f_{\mathrm{LO}}=1.8432 \mathrm{MHz}\right)$. The balanced output of the interferometer provides a signal, which after demodulation at $f_{\mathrm{LO}}$ can be used to lock the interferometer to a 'dark fringe' and to operate the apparatus as an active null instrument. The servo-signal is demodulated by a dual-phase lock-in amplifier at $f_{\mathrm{CP}}$ to reveal the phase shift signal. The loss signal is deduced from the low-frequency component of the balanced output signal by demodulation via a second dual-phase lock-in amplifier. FS: frequency shifter (180 $\mathrm{MHz}$.

$f_{\text {TLO }}<2 \mathrm{GHz}$ (TLO). Since it will be important in the discussion of the experimental results, we will focus for a moment on the experimental definition of the optical field frequencies. The servo-loops applied to the lasers determine the test field frequency, as seen by the atoms of the atomic beam providing the sample in our experiment (Fig. 2): $\nu_{\mathrm{TEST}}=f_{\mathrm{TLO}}-$ $f_{\mathrm{FS}}+\nu_{\mathrm{DRIVE}}^{\mathrm{T}}$. Here, $\nu_{\mathrm{DRIVE}}^{\mathrm{T}}$ gives the driving field frequency as it would appear for the atoms of the atomic beam, if the driving field and the test field were propagating in an exactly collinear way. Even if the driving laser is locked to the atomic resonance $\nu_{0}, \nu_{\text {DRIVE }}^{\mathrm{T}}$ can differ from $\nu_{0}$ if the test field does not intersect the atomic beam at right angle or if some residual frequency error (offset) is present in the FMS servo-loop. Hence, we may write

$\nu_{\mathrm{TEST}}=\Delta f_{\mathrm{TEST}}-\delta \nu_{\mathrm{TEST}}+\nu_{0}$, where $\Delta f_{\mathrm{TEST}}=f_{\mathrm{TLO}}-f_{\mathrm{FS}}$ and $\delta \nu_{\mathrm{TEST}}=\nu_{0}-$ $\nu_{\text {DRIVE }}^{\mathrm{T}}$. The detuning $\delta \nu_{\mathrm{TEST}}$ can be determined experimentally: for the optimum case $\delta \nu_{\mathrm{TEST}}=0$, the test field is resonant for $\Delta f_{\mathrm{TEST}}=0$, whereas for non-vanishing $\delta \nu_{\text {TEST }}$ the resonance will be shifted to $\Delta f_{\mathrm{TEST}}=\delta \nu_{\mathrm{TEST}}$. Further, we would like to emphasize that $\nu_{\mathrm{DRIVE}}^{\mathrm{T}}$ will differ from the frequency

$\nu_{\text {DRIVE }}=\Delta f_{\text {DRIVE }}-\delta \nu_{\text {TEST }}+\nu_{0}$

of the driving field actually seen by the atoms of the atomic beam, if test and driving field are not propagating exactly in collinear manner. During the experiment, $f_{\text {TLO }}$ is swept and absorption and phase shift spectra are recorded as a function of $\Delta f_{\text {TEST }}$. Hence, the driving field can be thought of to be located at $\Delta f_{\text {DRIVE }}$ within these spectra. This field appears to be resonant for the atoms of the atomic beam, if $\Delta f_{\text {DRIVE }}=\Delta f_{\text {TEST }}$ which is clear from (5).

To measure absorption and phase shift spectra, the probe beam enters a Mach-Zehnder interferometer (Fig. 2). A small fraction of the probe power is directed into the test arm and intersects a chopped atomic beam $\left(f_{\mathrm{CP}} \approx 1770 \mathrm{~Hz}\right)$ at right angle. Most of the probe power is directed into the reference arm, where the optical field is phase modulated $\left(f_{\mathrm{LO}}=\right.$ $1.8432 \mathrm{MHz}$ ). The balanced output of the interferometer provides a signal, which, after demodulation at $f_{\mathrm{LO}}$, can be used as an error signal for locking the interferometer to a 'dark fringe'. This way the apparatus can be operated as an active null instrument. The feedback signal of the corresponding servo-loop is applied to a piezo electric transducer not shown in the figure and to the phase modulator in the reference arm (electro-optic phase modulator, EOM). It also contains the information about the phase shift imposed on the test field during the interaction with the atoms of the chopped beam. Hence, detection of the servo-signal with a dual-phase lock-in amplifier reveals the phase shift of the optical test field. The absorption signal can be deduced from the balanced output of the interferometer: either the signal demodulated at $2 f_{\mathrm{LO}}$ or the low-frequency component of the balanced output itself may be read as well by a dual-phase lock-in amplifier demodulating at $f_{\mathrm{CP}}$. For the sake of experimental simplicity the latter situation was chosen for our experiment.

The novel spectroscopic method described here provides some important advantages when compared 
to the FM-spectroscopy, to the 'bare' Mach-Zehnder interferometer [23] or to the heterodyne interferometer [24], which have so far been used for the simultaneous measurement of absorption and phase shift (index of refraction) spectra. By spatially separating test and reference fields, this method intrinsically avoids problems which can arise from the unwanted interaction of the reference field with the sample and which could modify the reference or alter the optical properties of the medium under investigation. Further, by implementing a strongly unbalanced input beam-splitter, the strong local oscillator concept, well known from FM-spectroscopy, can be applied in order to achieve a good signal-to-noise ratio even in those cases where the test field has to be very weak. Finally, cross-talk between the absorption and phase shift signal as well as non-linearities arising at large phase shifts can be avoided, since the apparatus can be operated as an active null instrument for phase shift detection. The main disadvantage of this setup as compared to FMS or to the heterodyne interferometer is common to all optical interferometers: interference of mechanical and acoustical noise from the environment strongly reduces the phase shift detection limit at low Fourier-frequencies. Therefore, the measurement has to be shifted from ' $\mathrm{dc}$ ' to frequencies where the noise from the environment can be suppressed by technical means. In our experiment the atomic beam is chopped mechanically at a frequency of $f_{\mathrm{CP}} \approx 1770 \mathrm{~Hz}$. The interferometer is acoustically decoupled from the environment by operating the complete apparatus in an UHV-chamber. For mechanical decoupling, the interferometer is carried by an intra-vacuum 2-stage isolation mount. A more detailed description of the interferometer can be found in [26].

In the current setup, the probe field entering the interferometer has a power of $\sim 180 \mu \mathrm{W}$ resulting in a power of $P_{\mathrm{TEST}}=22 \mu \mathrm{W}$ at the interaction zone. The probe beam is slightly astigmatic and elliptical, with a beam waist of $w_{\text {hor }}=135 \mu \mathrm{m}$ and $w_{\text {vert }}=133 \mu \mathrm{m}$ at the interaction zone in the horizontal and vertical plane, respectively. The foci in the horizontal and vertical planes are located at a distance of $\Delta z_{\text {hor }} / b_{\text {hor }}=4 \times 10^{-4}$ and $\Delta z_{\text {vert }} / b_{\text {vert }}$ $=9.4 \times 10^{-2}$ from the interaction zone, where $b_{\text {hor }}$ $=0.27 \mathrm{~m}$ and $b_{\text {vert }}=0.25 \mathrm{~m}$ are the confocal parameters of the probe beam in the horizontal and vertical plane, respectively. Power and area of the test beam correspond to an intensity of $I_{\mathrm{TEST}}=0.40 \mathrm{~mW} / \mathrm{mm}^{2}$, which is a third of the saturation intensity [25]. Hence, saturation effects due to the test field are not expected to be present. The driving field enters the setup from the second port of the input beam-splitter. The test and driving fields both propagate in the same direction, slightly diverging by less than 20 $\mathrm{mrad}$. The linear polarization of each of the fields is oriented in the horizontal plane. At the interaction zone, the driving field has a beam waist of $w_{\text {hor }}=308$ $\mu \mathrm{m}$ in the horizontal and $w_{\text {vert }}=366 \mu \mathrm{m}$ in the vertical plane, with the foci located at a distance of $\Delta z_{\text {hor }} / b_{\text {hor }}=0.18$ and $\Delta z_{\text {vert }} / b_{\text {vert }}=-0.015$ from the interaction zone, where $b_{\text {hor }}=1.25 \mathrm{~m}$ and $b_{\text {vert }}$ $=1.99 \mathrm{~m}$ are the corresponding confocal parameters of the driving field. Hence we have ensured that the driving field cross sectional area exceeds the test field cross sectional area by a factor of $\sim 6$, so that the driving field intensity may be roughly assumed to be constant across the interaction zone. The maximum driving field power available at the interaction zone is about $P_{\text {DRIVE }}=10 \mathrm{~mW}$, which corresponds to a saturation parameter of $S=I / I_{\mathrm{SAT}}=(2 \Omega / \gamma)^{2}$ $=23$. The chopped atomic beam has a diameter of $l_{\mathrm{AB}} \approx 2 \mathrm{~mm}$ at the interaction zone, with a collimation of 1:200. Hence, at a typical oven temperature of $T \approx 1200 \mathrm{~K}$, the residual transversal Dopplerwidth $\left(\Delta \omega_{\mathrm{D}} \approx 0.2 \gamma\right)$ of the atoms is reduced to a value well below the natural linewidth.

So far, the phase shift detection limit has been determined by residual laser frequency noise. For an integration time of $1 / \Delta f_{\mathrm{BW}}=1 \mathrm{~s}$, a phase shift of $\varphi_{m}=1.2 \times 10^{-5} \mathrm{rad}$ can be detected with $\mathrm{SNR}=1$ [26]. Loss-detection is limited by residual laser power noise to a value of $\Delta P_{\mathrm{TEST}} / P_{\mathrm{TEST}}=2 \times 10^{-5}$ for an integration time of $1 / \Delta f_{\mathrm{BW}}=1 \mathrm{~s}$ [26]. Actually, an integration time of $1 \mathrm{~s}$ was chosen for the experiment.

In order to realize negative dispersive transparent media, we have recorded absorption and phase shift (index of refraction) spectra on the Ca-atomic beam for different driving field Rabi-frequencies $\Omega$. From each of these measurements, absorption and dispersion at bare atomic resonance $\Delta f_{\mathrm{TEST}}=\delta \nu_{\mathrm{TEST}}$ have been determined. These values can be compared to the prediction of the model (s. Eqs. (1) and (2)), which tells us that negative dispersion without ab- 


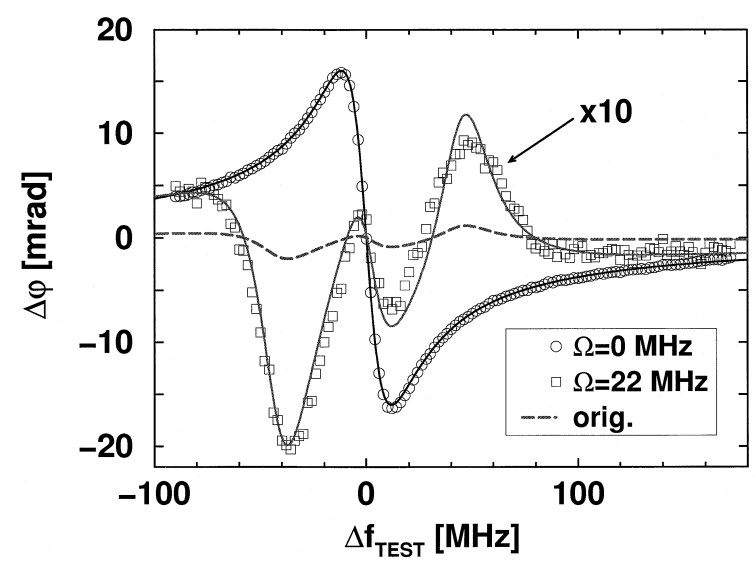

Fig. 3. Phase shift ( $\alpha$ index of refraction) versus probe detuning $\Delta f_{\mathrm{TEST}}$ for no driving $\Omega=0$ and strong driving $\Omega \sim 22 \mathrm{MHz}$. The solid lines correspond to a fit of the TLA-model to the experimental spectra. An interaction length of $l_{\mathrm{AB}}=2 \mathrm{~mm}$ is assumed. For no driving, the parameters are $N=7.513(17) \times 10^{8}$ $\mathrm{cm}^{-3}, \delta \nu_{\mathrm{TEST}}=-32(26) \mathrm{kHz}$ and $\Delta \nu_{0}=23.701(78) \mathrm{MHz}$. For the driving case we find $N=6.76(18) \times 10^{8} \mathrm{~cm}^{-3}, \delta \nu_{\text {TEST }}=$ $-2.58(54) \mathrm{MHz}, \Delta \nu_{0}=20.84(75) \mathrm{MHz}, \Omega=(2 \pi) 21.85(15)$ $\mathrm{MHz}$ and $\Delta \nu_{\text {DRIVE }}=1.60(37) \mathrm{MHz}$. For the driven atoms, the theoretical and experimental data have been multiplied by a factor of 10. For comparison, the original theoretical curve is also shown (orig). The errors given are purely statistical. The driving field Rabi-frequency corresponds to a saturation parameter of $S=4.39$.

sorption can be achieved in the regime of strong driving.

As an example, the experimental spectra corresponding to driving field Rabi-frequencies of $\Omega=0$ $\mathrm{MHz}$ and $\Omega \approx 22 \mathrm{MHz}$ are shown in Fig. 3 and Fig. 4. For the case of driving the spectra have additionally been given enlarged by a factor of 10 for the sake of clarity. The Rabi-sidebands located at detunings $\Delta f_{\text {TEST }} \sim 50 \mathrm{MHz}$ are clearly resolved, as well as the structure of the central resonance, which is of major importance for our purpose. From Fig. 4 it follows that the atoms exhibit gain without inversion $\left(\alpha l_{\mathrm{AB}}<0\right)$ in the vicinity of the bare atomic resonance [19]. More importantly, we find from the inspection of Fig. 3 and Fig. 4, that the suppression of resonant absorption is substantially stronger than that of resonant dispersion. Hence, we have clearly demonstrated experimentally that absorption can be suppressed while negative dispersion still is maintained. Furthermore, to provide a quantitative discussion of the experimental results, we have fitted the TLA-model $[15,16,21]$ to the experimental data.
These theoretical curves are shown as solid lines in Fig. 3 and Fig. 4. As fit parameters we chose the atomic density $N$, the natural linewidth $\Delta \nu_{0}=$ $\gamma /(2 \pi)$, the detuning $\delta \nu_{\mathrm{TEST}}$ of the bare atomic resonance from its nominal value (s. Eq. (4)), the driving field Rabi-frequency $\Omega$, and the detuning $\delta \nu_{\text {DRIVE }}$ of the driving field from the nominal bare atomic resonance (s. Eq. (5)). The results of the fits are given in the captions of Fig. 3 and Fig. 4. Firstly, we find that the observed linewidth $\overline{\Delta \nu_{0}}=22.0 \pm 1.0$ $\mathrm{MHz}$ is significantly smaller than the value for the natural linewidth found in the literature $\Delta \nu_{0, \text { LIT }}=34$ $\mathrm{MHz}$ [25] but is in good agreement with the experimental result of Hiller [27], who has measured the variation of the lifetime of the $4 \mathrm{~s} 4 \mathrm{p}^{1} \mathrm{P}_{1}$-level with Ca-vapor pressure. This reduction of linewidth is attributed to radiation trapping effects which play a role at atomic densities, atomic velocities, and spatial dimensions typical in our experiment [27,28]. Second, we notice that the atomic densities independently derived from the absorption and phase shift data agree reasonably well, with a difference not

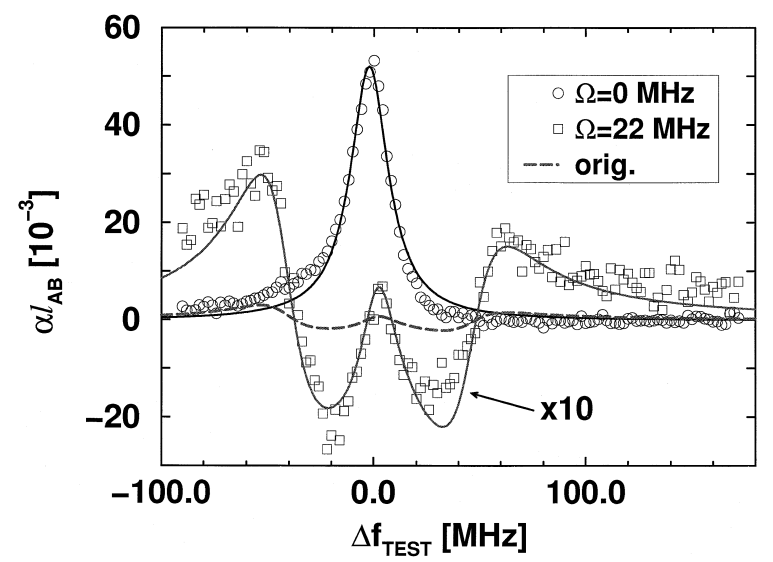

Fig. 4. Absorption $\left(\alpha l_{\mathrm{AB}}\right)$ versus probe detuning $\Delta f_{\mathrm{TEST}}$ for no driving $\Omega=0$ and strong driving $\Omega \sim 22 \mathrm{MHz}$. The solid lines correspond to a fit of the TLA-model to the experimental spectra. An interaction length of $l_{\mathrm{AB}}=2 \mathrm{~mm}$ is assumed. For no driving, these parameters are $N=6.12(11) \times 10^{8} \mathrm{~cm}^{-3}, \quad \delta \nu_{\text {TEST }}=$ $-2.27(20) \mathrm{MHz}$ and $\Delta \nu_{0}=22.10(65) \mathrm{MHz}$. For the driving case we find $N=6.91(28) \times 10^{8} \mathrm{~cm}^{-3}, \delta \nu_{\mathrm{TEST}}=-1.19(71) \mathrm{MHz}$, $\Delta \nu_{0}=21.6(14) \mathrm{MHz}, \Omega=(2 \pi) 22.62(20) \mathrm{MHz}$ and $\Delta \nu_{\text {DRIVE }}=$ $1.50(51) \mathrm{MHz}$. For the driven atoms, the theoretical and experimental data have been multiplied by a factor of 10 . For comparison, the original theoretical curve is also shown (orig). The errors given are purely statistical. The driving field Rabi-frequency corresponds to a saturation parameter of $S=4.39$. 
exceeding $23 \%$. Finally, we would like to point out that the determination of the driving field intensity from the spectra is surprisingly accurate $(\Delta \Omega / \bar{\Omega}=$ 0.035). This is due to the fact that on one hand there is only small correlation between $\Omega$ and the remaining parameters of the fit, but, on the other, this is not the case among the remaining parameters. This is to be expected, since $\Omega$ is the only quantity determining the 'qualitative shape' of the spectra in the case of strong driving, when the Rabi-sidebands are clearly resolved.

From the measurements performed at different Rabi-frequencies we have determined the absorption and dispersion at resonance. For some of the spectra, the driving field was slightly detuned $\left(\left|\delta \nu_{\text {DRIVE }} / \Delta \nu_{0}\right|<0.25\right)$ from bare atomic resonance, so that - to first order - the spectra were shifted in frequency. In order to account for this, we have determined the absorption and dispersion at $\Delta f_{\mathrm{TEST}}=\delta \nu_{\mathrm{DRIVE}}$ rather than at $\delta \nu_{\mathrm{TEST}}$. The result is given in Fig. 5, where the theoretical curves are shown for comparison. From this graph, we conclude

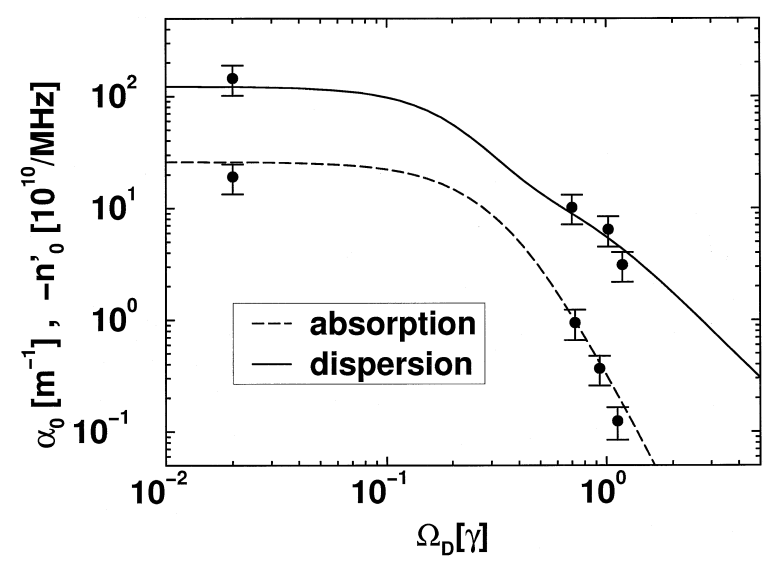

Fig. 5. Absorption $\alpha_{0}$ and dispersion $n_{0}^{\prime}=\partial_{\omega} n_{0}$ at bare atomic resonance versus driving field Rabi-frequency $\Omega$. For the theoretical curves the mean atomic density $\bar{N}=6.0 \times 10^{8} \mathrm{~cm}^{-3}$ was used, which was calculated from the spectra taken without driving. The corresponding experimental points are given at $\Omega=$ $0.02 \gamma$. All data points were derived from the theoretical spectra fitted to the experimental curves. Dispersion and absorption were taken at $\Delta f_{\mathrm{TEST}}=\delta \nu_{\mathrm{DRIVE}}$ rather than at $\Delta f_{\mathrm{TEST}}=\delta \nu_{\mathrm{TEST}}$. This is reasonable, since for small detunings of the driving field, the spectra are simply shifted to first order by $\delta \nu_{\text {DRIVE }}$. The experimental points have not been recalibrated with respect to the atomic density $N$, but the atomic densities corresponding to each single spectrum have been used. that we have reached a regime of driving intensities, where the suppression of absorption overcomes the suppression of dispersion. Therefore, we have in principle demonstrated experimentally that negative dispersive transparent media can be realized.

From Fig. 5 we conclude that a dispersion of $\partial_{\omega} n_{0}-(3.1 \pm 1.0) \times 10^{-10} / \mathrm{MHz}$ has been achieved at a residual absorption of $\alpha_{0}=(0.125 \pm$ $0.040) \% / \mathrm{cm}$ for an atomic density of $N=0.60 \times$ $10^{9} \mathrm{~cm}^{-3}$ and a saturation parameter of $S=$ $(2 \Omega / \gamma)^{2}=4.4$. The dispersion necessary to realize, for example, a white-light cavity at the Ca-wavelength is $\partial_{\omega} n_{0}=-1 / \omega_{0}=-2.2 \times 10^{-10} / \mathrm{MHz}$, which is somewhat less than the dispersion demonstrated experimentally. Hence, we have achieved a figure of merit corresponding to $\Theta_{\mathrm{FOM}}=11 \mathrm{~m}$. Without driving the atoms, a dispersion of $\partial_{\omega} n_{0}=$ $-124 \times 10^{-10} / \mathrm{MHz}$ would have been achieved at an absorption of $\alpha_{0}=2.6 \% / \mathrm{cm}$ which would correspond to a figure of merit of $\Theta_{\mathrm{FOM}}=2.2 \mathrm{~m}$. Hence, we have reduced the absorption by a factor of 208 . However, the figure of merit has only been increased by a factor of 5.5 due to the accompanying reduction of the dispersion.

Finally, we would like to relate our experimental results to the requirements which must be fulfilled for the realization of a white-light cavity. As an example, we focus on a cavity with length $l_{\mathrm{CAV}}=$ $1 \mathrm{~cm}$ and an (empty) finesse of $\mathscr{F}=64 \times 10^{3}$. We will assume that a sample of Ca-atoms is placed inside this cavity at a density of $N=5 \times 10^{10} \mathrm{~cm}^{-3}$. It is supposed that the atoms are driven by a resonant driving field of Rabi-frequency $\Omega=13.5 \gamma$ corresponding to a saturation parameter of $S \approx 730$. The linewidth of the cavity would then be increased by a factor of 12, resulting in a FWHM-linewidth of $\Delta \omega_{\mathrm{WLC}}=17.7 \mathrm{MHz}$ compared to $\Delta \omega_{\mathrm{CAV}}=$ $1.47 \mathrm{MHz}$ of the empty cavity. A single resonance of this cavity is shown in Fig. 6. From this we conclude that for the example system discussed here, it should be possible to increase the linewidth without significantly deteriorating the power build-up of the cavity. However, the situation related to this example corresponds to a figure of merit of the order of $\Theta_{\mathrm{FOM}} \approx$ $1000 \mathrm{~m}$, which exceeds the value realized experimentally by about a factor of 100 . This of course means that the experimental conditions have to be strongly improved to reach a regime where a white- 


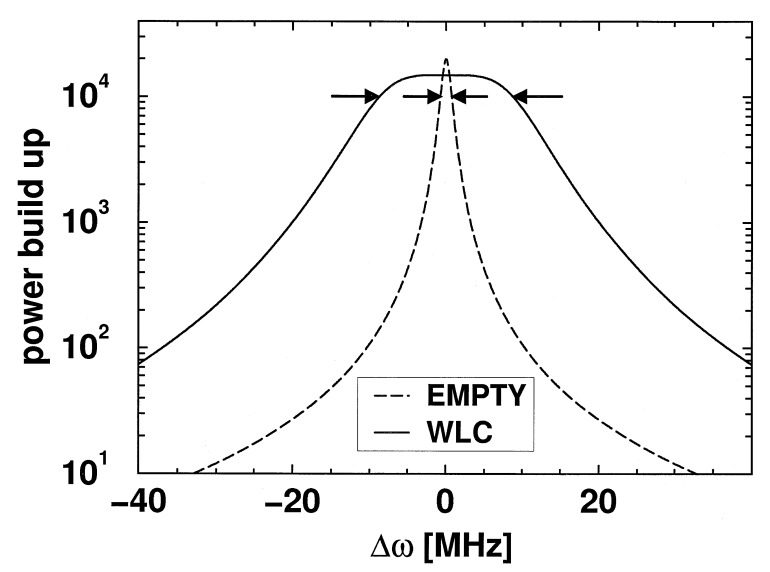

Fig. 6. Power build-up of an optical cavity in the vicinity of a single resonance versus detuning from resonance for (a) an intracavity sample of Ca-atoms (white-light cavity, WLC) and (b) an empty cavity (EMPTY). A cavity length of $l_{\mathrm{CAV}}=1 \mathrm{~cm}$ and a finesse of $\mathscr{F}=64 \times 10^{3}$ is assumed. $N=5 \times 10^{10} \mathrm{~cm}^{-3}, \gamma=$ $34 \mathrm{MHz}$, and driving field Rabi-frequency $\Omega=13.5 \gamma$. For the empty cavity the FWHM-linewidth is $\Delta \omega=1.47 \mathrm{MHz}$, while for the white-light cavity we find $\Delta \omega=17.7 \mathrm{MHz}$.

light cavity may be feasible experimentally. Although the dispersion achieved so far exceeds the value which is required, the absorption has to be reduced by about 4 orders of magnitude. This could be achieved by strongly enhancing the driving field intensity by about 2 orders of magnitude. We would then find that the dispersion has also been reduced, so that an atomic density 100 times the density used in our experiment would be necessary to compensate for this reduction.

In conclusion, we have demonstrated experimentally that negative dispersion without absorption can be realized. In the strongly driven atoms of a Caatomic beam, a dispersion of $\partial_{\omega} n_{0}=-(3.1 \pm 1.0) \times$ $10^{-10} / \mathrm{MHz}$ accompanied by a residual absorption of $\alpha_{0}=0.125 \pm 0.040 \% / \mathrm{cm}$ has been measured. This corresponds to a figure of merit of $\Theta_{\mathrm{FOM}}=11$ $\mathrm{m}$. This value has to be compared to $\Theta_{\mathrm{FOM}} \sim 10^{3} \mathrm{~m}$, which has to be achieved for certain applications of negative dispersive transparent media. For future experiments we will therefore improve our atomic beam source. Modest enhancement of driving field power can be achieved by tighter focusing of the driving beam. However, in order to increase the driving field intensity by orders of magnitude, high power laser systems or a resonant enhancement of the driving field must be used. The first experimental realization of a white-light cavity should then be possible.

\section{Acknowledgements}

This work was supported by the Sonderforschungsbereich SFB 407 of the Deutsche Forschungsgemeindschaft DFG.

\section{References}

[1] B.S. Ham, P.R. Hemmer, M.S. Shahriar, Opt. Commun. 144 (1997) 227.

[2] O. Kocharovskaya, Y.I. Khanin, Pis'ma Zh. Eksp. Teor. Fiz. 48 (1988) 581 [JETP Lett. 48 (1988) 630].

[3] S.E. Harris, Phys. Rev. Lett. 62 (1989) 1033.

[4] M.O. Scully, S.Y. Zhu, A. Gavrielides, Phys. Rev. Lett. 62 (1989) 2813.

[5] M.O. Scully, S.Y. Zhu, H. Fearn, Z. Phys. D 22 (1992) 471; S.Y. Zhu, M.O. Scully, H. Fearn, L.M. Narducci, Z. Phys. D 22 (1992) 483; H. Fearn, M.O. Scully, S.Y. Zhu, M. Sargent III, Z. Phys. D 22 (1992) 495; for an overview, see the topical issue of Quantum Optics 6 (4) (1994).

[6] A. Nottelmann, C. Peters, W. Lange, Phys. Rev. Lett. 70 (1993) 1783; W.E. van der Veer, R.J.J. van Diest, A. Dönszelmann, H.B. van Linden van den Heuvell, Phys. Rev. Lett. 70 (1993) 3243; E.S. Fry, X. Li, D. Nikonov, G.G. Padmabandu, M.O. Scully, A.V. Smith, F.K. Tittel, C. Wang, S.R. Wilkinson, S.-Y. Zhu, Phys. Rev. Lett. 70 (1993) 3235; G.G. Padmabandu, G.R. Welch, I.N. Shubin, E.S. Fry, D.E. Nikonov, M.D. Lukin, M.O. Scully, Phys. Rev. Lett. 76 (1996) 2053.

[7] O. Kocharovskaya, Y. Rostovstev, in: IQEC, vol. 7, 1998, OSA Technical Digest Series (Optical Society of America, Washington DC, 1998), pp. 141-142.

[8] M. Fleischhauer, C.H. Keitel, M.O. Scully, C. Su, B.T. Ulrich, S.-Y. Zhu, Phys. Rev. A 46 (1992) 1468; H. Friedmann, A.D. Wilson-Gordon, Opt. Commun. 98 (1993) 303; U. Rathe, M. Fleischhauer, S.-Y. Zhu, T.W. Hänsch, M.O. Scully, Phys. Rev. A 47 (1993) 4994.

[9] M. Xiao, Y. Li, S. Jin, J. Gea-Banacloche, Phys. Rev. Lett. 74 (1995) 666; A.S. Zibrov, M.D. Lukin, L. Hollberg, D.E. Nikonov, M.O. Scully, H.G. Robinson, V.L. Velichansky, Phys. Rev. Lett. 76 (1996) 3935.

[10] M.O. Scully, Phys. Rep. 219 (1992) 191.

[11] L.V. Hau, S.E. Harris, Z. Dutton, C.H. Behroozi, Nature 397 (1999) 594.

[12] H. Lee, M. Fleischhauer, M.O. Scully, Phys. Rev. A 58 (1998) 2587; R. Wynands, A. Nagel, Appl. Phys. B 68 (1999) 1.

[13] G. Müller, M. Müller, A. Wicht, R.-H. Rinkleff, K. Danzmann, Phys. Rev. A. 56 (1997) 2385. 
[14] A. Wicht, K. Danzmann, M. Fleischhauer, M. Scully, G. Müller, R.-H. Rinkleff, Opt. Commun. 134 (1997) 431

[15] C. Szymanowski, A. Wicht, K. Danzmann, J. Mod. Opt. 44 (1997) 1373.

[16] A. Wicht, Ph.D. Thesis, Universität Hannover, Germany, 1998.

[17] S.G. Rautian, Sobel'man, Soviet Phys. JETP 14 (1962) 328.

[18] B.R. Mollow, Phys. Rev. 188 (1969) 1969.

[19] W. Hartig, H. Walther, Appl. Phys. 1 (1973) 171; F.Y. Wu, S. Ezekiel, M. Ducloy, B.R. Mollow, Phys. Rev. Lett. 38 (1977) 1077; C. Wei, N.B. Manson, Phys. Rev. A 49 (1994) 4751; for further references related to the subject of strong dispersion without absorption in two-level schemes see [15].

[20] Th. Kisters, K. Zeiske, F. Riehle, J. Helmcke, Appl. Phys. B 59 (1994) 89.
[21] H. Friedmann, A.D. Wilson-Gordon, Phys. Rev. A 36 (1987) 1333.

[22] O.S. Brozek, V. Quetschke, A. Wicht, K. Danzmann, Opt. Commun. 146 (1998) 141.

[23] O. Schmidt, R. Wynands, Z. Hussein, D. Meschede, Phys. Rev. A 53 (1996) R27.

[24] G. Müller, A. Wicht, R.-H. Rinkleff, K. Danzmann, Opt. Commun. 127 (1996) 37.

[25] A. Witte, Th. Kisters, F. Riehle, J. Helmcke, J. Opt. Soc. Am. B 9 (1992) 1030.

[26] A. Wicht, M. Müller, V. Quetschke, R.-H. Rinkleff, A. Rocco, K. Danzmann, submitted for publication.

[27] W. Hiller, Diploma-Thesis, Universität Hannover, Germany, 1980.

[28] B.P. Kibble, G. Copley, L. Krause, Phys. Rev. 153 (1967) 9. 